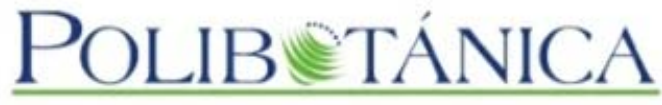

\title{
Polibotánica
}

ISSN electrónico: 2395-9525

polibotanica@gmail.com

Instituto Politécnico Nacional

México

http://www.polibotanica.mx

\section{CONHECIMENTO BOTÂNICO LOCAL DE AGRICULTORES DO SEMIÁRIDO DO ESTADO DA PARAIÍBA, NORDESTE DO BRASIL}

\author{
CONOCIMIENTO BOTÁNICO LOCAL DE \\ AGRICULTORES DEL SEMIÁRIDO DEL \\ ESTADO DE LA PARAÍBA, NORDESTE DEL \\ BRASIL
}

\section{LOCAL BOTANICAL KNOWLEDGE OF FARMERS OF THE SEMIARID OF PARAIIBA STATE, NORTHEAST OF BRAZIL}

Barbosa, E. U. G., T.K.N. Carvalho, E. C. Ferreira, S. S. Santos y R. F. P. Lucena CONHECIMENTO BOTÂNICO LOCAL DE AGRICULTORES DO SEMIÁRIDO DO ESTADO DA PARAÍBA, NORDESTE DO BRASIL

CONOCIMIENTO BOTÁNICO LOCAL DE AGRICULTORES DEL SEMIÁRIDO DEL ESTADO DE LA PARAÍBA, NORDESTE DEL BRASIL

LOCAL BOTANICAL KNOWLEDGE OF FARMERS OF THE SEMIARID OF PARAIIBA STATE, NORTHEAST OF BRAZIL

POLIBETÁNICA

Instituto Politécnico Nacional
Núm. 50: 191-208 México. Agosto 2020

DOI: $10.18387 /$ polibotanica.50.13

(c) Este es un artículo de acceso abierto bajo la licencia Creative Commons 4.0 Atribución-No Comercial (CC BY-NC 4.0 Internacional). 
CONHECIMENTO BOTÂNICO LOCAL DE AGRICULTORES DO SEMIÁRIDO DO ESTADO DA PARAÍBA, NORDESTE DO BRASIL

\author{
CONOCIMIENTO BOTÁNICO LOCAL DE AGRICULTORES DEL SEMIÁRIDO \\ DEL ESTADO DE LA PARAÍBA, NORDESTE DEL BRASIL
}

\title{
LOCAL BOTANICAL KNOWLEDGE OF FARMERS OF THE SEMIARID OF PARAÍBA STATE, NORTHEAST OF BRAZIL
}

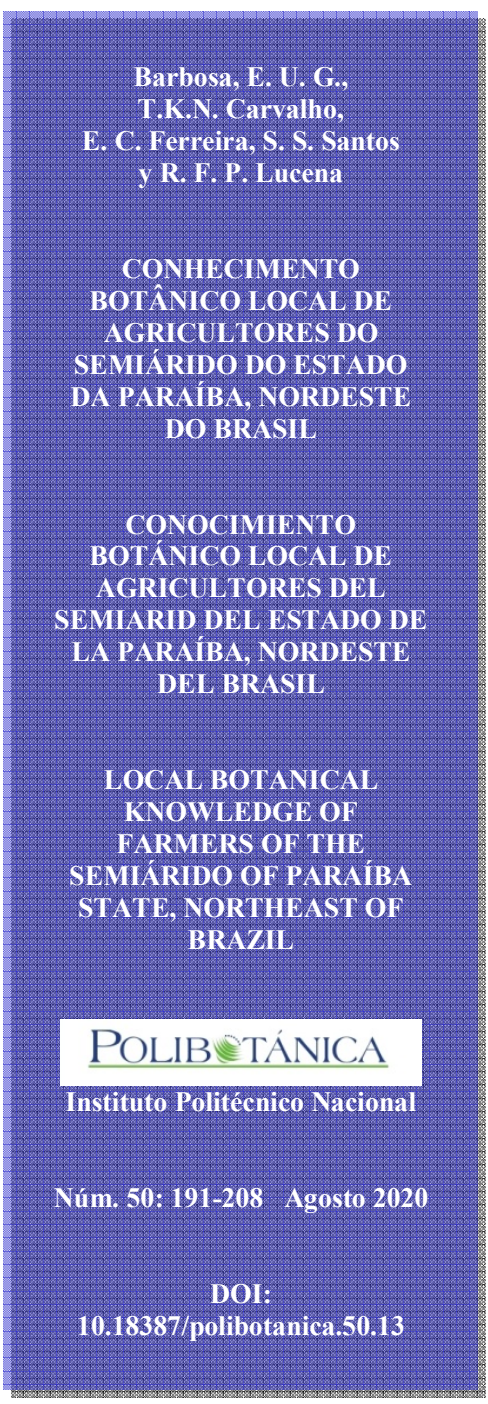

\author{
E. U. G. Barbosa \\ T.K.N. Carvalho \\ E. C. Ferreira \\ S. S. Santos \\ R. F. P. Lucena / rlucena@dse.ufpb.br \\ Programa de Pós-Graduação em Desenvolvimento e Meio Ambiente. Laboratório de \\ Etnobiologia e Ciências Ambientais. Centro de Ciências Exatas e da Natureza. \\ Universidade Federal da Paraíba, Campus I, João Pessoa, Paraíba, Brasil.
}

RESUMO: Diversos estudos desenvolvidos no estado da Paraíba, Nordeste do Brasil, descrevem a forma como as populações rurais da Caatinga interagem com plantas lenhosas úteis disponíveis na região. Nesse contexto, o presente estudo descreve e analisa a inter-relação de moradores da comunidade rural de Santa Rita, no Cariri da Paraíba, com as plantas lenhosas úteis, avaliando sua disponibilidade por meio de inventário etnobotânico e da vegetação. A pesquisa foi conduzida no Município do Congo, e os dados foram coletados entre os anos de 2011 e 2012, com entrevista semiestruturada, realizada com os chefes (homem e mulher) de cada residência. Os dados foram analisados por meio do valor do uso. Para amostragem fitossociológica foram registrados todos os indivíduos arbustivo-arbóreos lenhosos vivos com DNS (diâmetro do caule a nível de solo) $\geq 3 \mathrm{~cm}$, se tomando nota também da sua altura estimada. Foram registradas 56 espécies vegetais úteis, destas, 42 identificadas, distribuídas em 37 gêneros e 20 famílias, sendo as mais citadas Myracrodruon urundeuva Allemão, Tabebuia aurea (Silva Manso) Benth. \& Hook. f. ex S. Moore, e Sideroxylon obtusifolium (Roem \& Schult.), T. D. Penn. As principais categorias de uso foram tecnologia, medicinal, combustível e construção. Em relação ao valor de uso (VU) as espécies de maior destaque foram T. aurea (Craibeira) ( $\mathrm{VU}=5,88)$, Aspidosperma. Pyrifolium Mart. (pereiro) $(3,74)$, e S. obtusifolium (quixabeira) $(3,71)$. $\mathrm{Na}$ fitossociologia foram registradas 24 espécies pertencentes a 21 gêneros e 12 famílias. Dos 2000 indivíduos amostrados, 55 não foram identificados. Destacaram-se as seguintes famílias botânicas: Euphorbiaceae, (1524 indivíduos), Fabaceae (271 indivíduos) e Apocynaceae (117 indivíduos). Entre as espécies, houve destaque para Croton blanchetianus Baill (marmeleiro), com 1400 indivíduos, seguido por Poincianella pyramidalis Tul. (catingueira) (198 indivíduos) e A. pyrifolium (pereiro) (117 indivíduos). O estudo indica que a composição da vegetação local na comunidade rural de Santa Rita é oriunda de altos níveis de antropização, sendo necessários maior detalhamento na dinâmica de uso e retirada dos indivíduos que demonstraram maior significado cultural e valor de uso.

Palavras-Chave: Etnobotânica, Comunidade rural, Caatinga. 
ABSTRACT: Several studies carried out in the state of Paraíba (Northeast Brazil) have described how rural populations interact with useful woody plants available in the Caatinga. In this context, this study aimed to describe and analyze the interrelationship between residents in the rural community of Santa Rita (Cariri of Paraíba) and useful woody plants, evaluating their availability through ethnobotanical and plant inventory. The research was conducted in the municipality of Congo, and the data were collected between 2011 and 2012 through semistructured interview with the householders (men and women). The data were analyzed using the use value. For phytosociological sampling, all woody shrub-tree individuals with DGL (stem diameter at ground level) $\geq 3 \mathrm{~cm}$ were recorded and their estimated height was also recorded. Fifty-six useful plant species were recorded, of which 42 were identified and distributed in 37 genera and 20 families. Myracrodruon urundeuva Allemão, Tabebuia aurea (Silva Manso) Benth. \& Hook. f. ex S. Moore, and Sideroxylon obtusifolium (Roem \& Schult.), T. D. Penn were the most cited species. Technology, medical, fuel, and construction were the main use categories. Regarding the Use Value (UV), T. aurea $(\mathrm{UV}=5.88)$, Aspidosperma. pyrifolium Mart. (pereiro) (3.74), and S. obtusifolium (quixabeira) (3.71) were the most prominent species. Twenty-four species belonging to 21 genera and 12 families were recorded in the phytosociology. Of the 2000 sampled individuals, 55 were not identified. The following botanical families stood out: Euphorbiaceae, (1524 individuals), Fabaceae (271 individuals), and Apocynaceae (117 individuals). Croton blanchetianus Baill (marmeleiro) (1400 individuals), Poincianella pyramidalis Tul. (catingueira) (198 individuals) and A. pyrifolium (pereiro) (117 individuals) were the most prominent species. This study indicates that the local vegetation composition in the rural community of Santa Rita results from high degrees of anthropization; therefore, it is required a detailed study on the dynamics of use and removal of individuals that showed the highest cultural significance and use value.

Key words: Ethnobotany, Rural community, Caatinga.

\section{INTRODUÇÃO}

A etnobiologia é uma ciência norteada, principalmente, pelo registro e entendimento do conhecimento e uso de populações tradicionais acerca do ambiente natural (Albuquerque, Medeiros, \& Casas, 2015). Nos dias atuais, já são estabelecidos diálogos que ampliam as metodologias específicas da área possibilitando, por exemplo, a compreensão do processo evolutivo que acompanhou a relação homem/ambiente, por meio de investigações sobre a evolução cultural e a etnobiologia evolutiva (Santoro, Nascimento, Soldati, Ferreira Júnior, \& Albuquerque, 2018).

A etnobotânica, subárea da etnobiologia, investiga a inter-relação de populações humanas com os recursos vegetais (Albuquerque \& Andrade, 2002), e por isso é considerada como instrumento fundamental para a compreensão da dinâmica da propriedade cultural e intelectual do conhecimento, o uso e a extração de espécies, podendo seus resultados contribuir para administração sustentável dos recursos naturais (Sousa, Silva, Rocha, Santana, \& Vieira, 2015).

No Brasil, é registrada uma maior concentração de pesquisadores desenvolvendo estudos etnobotânicos na Mata Atlântica (região Sul e Sudeste), e na Caatinga (região Nordeste), principalmente nos estados da Paraíba e Pernambuco, podendo ser reflexo da rica sociodiversidade, das complexas formações vegetacionais, e da maior concentração de grupos de pesquisas nestas áreas. O direcionamento das pesquisas nestes locais tem contribuído para elucidar a dinâmica desta inter-relação e uso de plantas medicinais e alimentares, por exemplo (Liporacci, Hanazaki, Ritter, \& Araújo, 2017).

Diversos estudos desenvolvidos no estado da Paraíba descrevem a forma como as populações rurais da Caatinga interagem com plantas lenhosas úteis em diferentes regiões (Carvalho et al., 2012; Leite et al., 2012; Lucena, Medeiros, Araújo, Alves, \& Albuquerque, 2012; N. Silva et al., 2014), investigam categorias de uso específicas (Coutinho et al., 2015; Marreiros, Ferreira, 
Lucena, \& Lucena, 2015), espécies e famílias botânicas de importância ecológica, cultural e econômica (Guerra et al., 2014; Lucena et al., 2014, 2015, 2013; Pedrosa et al., 2015).

A Caatinga se estende por $912,529 \mathrm{Km}^{2}$, que ocupam partes de todos os estados do Nordeste do Brasil e região norte de Minas Gerais, e apresentam o habitat perfeito para abrigar sistemas sócio-ecológicos complexos. São registradas 5,218 espécies de plantas (Forzza et al., 2012) e este número tende a aumentar proporcionalmente ao número de estudos direcionados a esta área. Também é a moradia de cerca de 28,6 milhões de pessoas, onde $36 \%$ deste total reside nas zonas rurais, desenvolvendo uma relação específica com os recursos naturais que os circundam (Silva, Leal, \& Tabarelli, 2017).

Esta relação dos habitantes de áreas rurais com os recursos naturais na Caatinga é marcada por diversos fatores, inclusive pelos agravos de conflitos socioambientais e climáticos, que refletem na antropização que já abrange $63 \%$ do território, e no processo de desertificação das zonas consideradas de moderado e alto risco (Torres, Lapola, \& Gamarra, 2017). Tais zonas já ocupam 94\% da área total do ecossistema (Silva et al., 2017). Estes aspectos somados aos recentes avanços das mudanças climáticas, caracterizam tanto o ecossistema quanto as populações tradicionais como agentes vulneráveis ao clima (IPCC : Summary for Policymakers, 2013).

Em ambientes como a Caatinga, a etnobotânica é considerada subsídio assertivo para que se comprenda a participação das populações tradicionais dentro do processo de conservação. Em um estudo etnobotânico abordando essa temática é explicado que na visão das populações tradicionais, a conservação é mais do que o cuidado para o não desaparecimento de espécies, e sim uma relação mais forte com todo o "funcionamento" do ecossistema para que prossiga garantindo o provimento de suas necessidades e manutenção de suas vidas (Pedrosa, Almeida, Ramos, Barboza, \& Lopes, 2019).

Para isso, as pesquisas etnobotânicas, há mais de duas décadas vem se aprimorando sobre métodos de quantificação da importância utilitária das espécies vegetais e como relacionar isso às condições de recursos vegetais disponíveis nas comunidades locais. Um exemplo é o valor de uso (VU) (Phillips \& Gentry, 1993a, 1993b) que é uma técnica quantiativa simples que pode ser aplicada em grande quatidade de dados etnobotânicos para avaliar a utilidade relativa das espécies no contexto do conhecimento tradicional, podendo auxiliar no teste de hipóteses, como exemplo a hipótese da aparência ecológica que testa se as espécies mais importantes localmente são as mais disponiveis na vegetação local; estabelecendo correlações entre o conhecimento tradicional e informações ambientais.

Portanto, o presente estudo tem por objetivo descrever e analisar a inter-relação de moradores da comunidade rural de Santa Rita, no estado da Paraíba, Nordeste do Brasil, com as plantas lenhosas úteis, estimando seu valor de uso e importância relativa local.

\section{Procedimentos metodológicos}

\section{Área de estudo}

O município do Congo está localizado na mesorregião da Borborema e microrregião do Cariri Ocidental (707 $47^{\prime}$ " S; 36³9'34" W), no semiárido do estado da Paraíba, Nordeste do Brasil (fig. 1). Está situado a cerca de $212 \mathrm{~km}$ da capital do estado, João Pessoa, e faz divisa com os municípios de Coxixola, Caraúbas, Camalaú e Sumé no estado da Paraíba e Santa Cruz do Capibaribe no estado de Pernambuco (Guerra et al., 2015). Tem população estimada de 4.789 habitantes em um território de $333,471 \mathrm{~km}^{2}$ (IBGE. Instituto Brasileiro de Geografia e Estatística, 2018). As médias de temperatura são superiores a $24^{\circ} \mathrm{C}$, entretanto, atinge temperaturas mais amenas (média de $15^{\circ} \mathrm{C}$ ) nos meses de junho e julho (Mendes, Chaves, \& Chaves, 2008a). O clima semiárido caracteriza as altas temperaturas e precipitação anual abaixo de $500 \mathrm{~mm}$ (Mendes, Chaves, \& Chaves, 2008b). 
A comunidade rural de Santa Rita, onde o estudo foi realizado está localizada a cerca de 8 quilômetros do centro do urbano. Está subdividida em duas localidades, sendo Santa Rita de Cima e Santa Rita de Baixo, que distam $7 \mathrm{~km}$ uma da outra. A economia tem base na agropecuária. Possui templo religioso da Igreja Católica e grupo escolar e transporte para os estudantes até o centro urbano. Os moradores são acompanhados por Agente Comunitário de Saúde e o abastecimento de água é realizado pelo próprio município (Guerra et al., 2015; Lucena et al., 2015).

A população local se caracteriza por se reconhecerem predominantemente como agricultores e pecuaristas tradicionais. Utilizam os recursos vegetais circundantes como matéria-prima para subsidiar grande parte da sua dinâmica de vida e estruturação de suas propriedades. Os recursos vegetais à sua volta estão presentes em fragmentos de mata distribuídos entre trechos de "capoeira" (caatinga aberta), mais encontrados em Santa Rita de Cima; e na Serra da Engabelada que é circundada pelas casas dos habitantes da localidade de Santa Rita de Baixo.

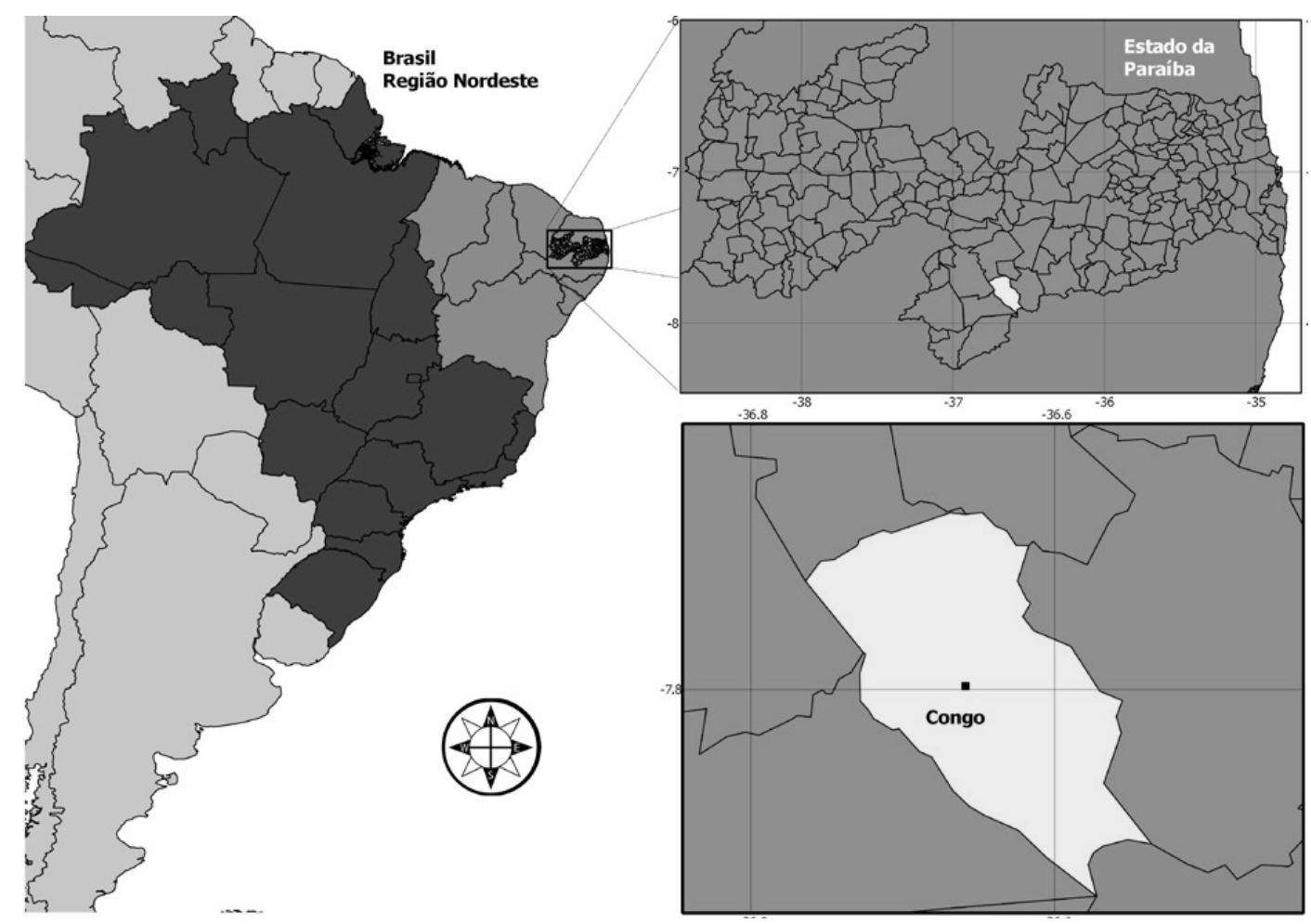

Fig. 1. Localização do município do Congo, na mesorregião da Borborema e microrregião do Cariri Ocidental, no semiárido do estado da Paraíba, Nordeste do Brasil. Mapa: (Guerra et al., 2015).

\section{Dados Etnobotânicos}

Esta pesquisa foi aprovada pelo Comitê de Ética em Pesquisa do Hospital Universitário Lauro Wanderley da Universidade Federal da Paraíba (CEP/HULW n ${ }^{\circ}$ 297/11, Folha de Rosto $n^{\circ}$ 420134).

Os dados foram coletados entre os anos de 2011 e 2012. O inventário etnobotânico foi realizado a partir de entrevista semiestruturada, realizada com os chefes (homem e mulher) de cada residência, mediante explicação do objetivo do estudo e assinatura do Termo de Consentimento Livre e Esclarecido. Foram visitadas 100\% das residências, excluindo da amostragem aquelas 
onde os chefes de família se negaram a participar da pesquisa. No total, foram entrevistados 98 informantes, sendo 41 homens com idade entre 22 e 87 anos, e 57 mulheres de 19 a 76 anos.

O formulário continha questionamentos sobre as espécies de plantas lenhosas nativas que os informantes conheciam e as suas formas de uso (R. F. P. Lucena et al., 2012). As plantas foram elencadas dentro de categorias utilitárias, seguindo a literatura específica da área (Albuquerque \& Andrade, 2002; R. F. P. Lucena et al., 2012). Também foram incorporadas informações provenientes de observação participante e turnê guiada, técnicas que auxiliam na confirmação dos dados das entrevistas e na identificação das espécies vegetais citadas (Albuquerque, Lucena, Alencar, 2010).

As espécies citadas foram organizadas de acordo com a sua forma de uso em categorias, nomeadas e adaptadas de acordo com literatura específica (Albuquerque \& Andrade, 2002, 2002; Lucena et al., 2017) Tais espécies foram coletadas, herborizadas, classificadas e tombadas na coleção do herbário Jaime Coêlho de Moraes (EAN).

A partir dos dados coletados nas entrevistas, foi calculado o valor de uso para as cada espécie, com a seguinte fórmula (Rossato, Leitão-Filho, \& Begossi, 1999), adaptada a partir de Phillips \& Gentry (1993a, 1993b).

$$
\mathrm{VU}=\frac{\sum \mathrm{Ui}}{\mathrm{n}}
$$

Onde, $\mathrm{Ui}=$ número de usos mencionados pelos informantes e $\mathrm{n}=$ número total de informantes.

\section{Dados da Vegetação}

O inventário da vegetação foi feito por meio do método de ponto quadrante, que consiste em fazer uma cruz com duas peças de madeira, onde cada lado representa um quadrante. Os quadrantes foram registrados em sentido horário, sendo amostrados os indivíduos mais próximos de cada quadrante (Araújo \& Ferraz, 2010). Os pontos foram lançados ao longo de transectos de 100 metros, sendo, no total, plotados 50 transectos aleatórios, com uma distância mínima de 10 metros entre cada ponto e de 10 metros entre cada transecto. Em cada transecto foram registrados 10 pontos, totalizando 500 pontos. Quarenta plantas foram registradas em cada transectos, totalizando 2000 plantas amostradas.

Para amostragem fitossociológica foram registrados os indivíduos arbustivo-arbóreos lenhosos vivos com DNS (diâmetro do caule a nível de solo) $\geq 3 \mathrm{~cm}$, tomando-se nota também da sua altura estimada. Cactos, bromélias, lianas e herbáceas foram excluídos da amostragem (Araújo \& Ferraz, 2010). Os índices analisados foram densidade relativa (DeR), dominância relativa (DoR), frequência relativa (FRt) e valor de importância (VI) (Araújo \& Ferraz, 2010), com o auxílio do software FITOPAC 2.1.As áreas amostrais foram selecionadas de acordo com a indicação dos informantes através de conversas informais. Foi pedido que indicassem as áreas de coleta de recurso. Excluiu-se dos resultados deste estudo as plantas que foram amostradas na fitossociologia, mas que não foram citadas pelos informantes.

\section{RESUltados E DisCUSSÃO}

\section{Inventário etnobotânico}

Registraram-se 56 espécies vegetais úteis, destas 42 foram identificadas, estando distribuídas em 37 gêneros e 20 famílias (Tabela 1). As espécies mais citadas foram Myracrodruon urundeuva Allemão (57 citações), Tabebuia aurea (Silva Manso) Benth. \& Hook. f. ex S. Moore (56 citações), e Sideroxylon obtusifolium (Roem \& Schult.), T. D. Penn (44 citações). Em relação às famílias botânicas, verificou-se que as mais representativas com relação ao 
número espécies foram Fabaceae (11 espécies), Euphorbiaceae (6 espécies), Anacardiaceae (3 espécies) e Bigoniaceae (3 espécies).

Tabela 1. Espécies e famílias registradas no levantamento etnobotânico e fitossociológico na comunidade Santa Rita, município do Congo, estado da Paraíba, Nordeste do Brasil, seguidas das categorias e partes usadas.

\begin{tabular}{|c|c|c|c|c|c|c|c|c|c|c|}
\hline Família / Espécie & Nome vernacular & Usos & $\begin{array}{l}\text { Partes das } \\
\text { plantas }\end{array}$ & $\mathbf{V U}$ & $\begin{array}{l}\mathrm{N}^{\circ} \\
\text { Ind }\end{array}$ & VI & DeR & DoR & FRt & $\begin{array}{l}\text { Área } \\
\text { basal }\end{array}$ \\
\hline \multicolumn{11}{|l|}{ Anacardiaceae } \\
\hline $\begin{array}{c}\text { Myracrodruon } \\
\text { urundeuva Allemão }\end{array}$ & Aroeira & $\begin{array}{l}\mathrm{Cb}, \mathrm{Ct}, \mathrm{Fr} \\
\mathrm{Me}, \mathrm{Ot}, \mathrm{Tc} \\
\mathrm{Vt}\end{array}$ & $\begin{array}{c}\mathrm{Ca}, \mathrm{Ec}, \mathrm{Fo} \\
\mathrm{Fr}, \mathrm{Ma}, \mathrm{Pc}, \mathrm{Se}\end{array}$ & 2,76 & 3 & 1,61 & 0,15 & 1,19 & 0,32 & 0,24 \\
\hline $\begin{array}{c}\text { Schinopsis } \\
\text { brasiliensis Engl. }\end{array}$ & Baraúna & $\begin{array}{l}\mathrm{Cb}, \mathrm{Ct}, \mathrm{Fr} \\
\mathrm{Me}, \mathrm{Or}, \mathrm{Ot} \\
\mathrm{Tc}, \mathrm{Vt}, \mathrm{Va}\end{array}$ & $\begin{array}{l}\mathrm{Ca}, \mathrm{Fl}, \mathrm{Fo}, \mathrm{Fr} \\
\mathrm{Ma}, \mathrm{Pc}, \mathrm{Se}\end{array}$ & 1,29 & 2 & 0,73 & 0,1 & 0,42 & 0,22 & 0,08 \\
\hline $\begin{array}{c}\text { Spondias tuberosa } \\
\text { Arruda }\end{array}$ & Umbuzeiro & $\begin{array}{l}\mathrm{Al}, \mathrm{Cb}, \mathrm{Fr} \\
\mathrm{Me}, \mathrm{Or}, \mathrm{Ot} \\
\text { Tc, Vt }\end{array}$ & $\begin{array}{c}\mathrm{Ca}, \mathrm{Ec}, \mathrm{Fo}, \\
\text { Fr, Ma, Pc, } \\
\text { Tb }\end{array}$ & 2,21 & 2 & 0,83 & 0,1 & 0,51 & 0,22 & 0,1 \\
\hline \multicolumn{11}{|l|}{ Apocynaceae } \\
\hline $\begin{array}{l}\text { Aspidosperma } \\
\text { pyrifolium Mart. }\end{array}$ & Pereiro & $\begin{array}{l}\mathrm{Cb}, \mathrm{Ct}, \mathrm{Fr} \\
\mathrm{Me}, \mathrm{Or}, \mathrm{Ot} \\
\mathrm{Tc}, \mathrm{Vt}, \mathrm{Va}\end{array}$ & $\begin{array}{l}\mathrm{Ca}, \mathrm{Ec}, \mathrm{Fl}, \\
\text { Fo, Ma, Pc, } \\
\mathrm{Ra}\end{array}$ & 3,74 & 117 & 19,3 & 5,85 & 2,88 & 10,6 & 0,59 \\
\hline \multicolumn{11}{|l|}{ Arecaceae } \\
\hline $\begin{array}{l}\text { Copernicia prunifera } \\
\text { (Miller) It.E.Moore }\end{array}$ & Carnaúba & $\mathrm{Ct}, \mathrm{Fr}, \mathrm{Tc}$ & Fr, Ma & 0,06 & - & - & - & - & - & - \\
\hline $\begin{array}{l}\text { Syagrus Oleracea } \\
\text { (Mart.) Becc }\end{array}$ & Côco catolé & $\mathrm{Al}, \mathrm{Fr}, \mathrm{Me}, \mathrm{Tc}$ & Fo, Fr, Ra & 0,17 & - & - & - & - & - & - \\
\hline Bignoniaceae & & & & & & & & & & \\
\hline $\begin{array}{c}\text { Tabebuia aurea } \\
\text { (Silva Manso) Benth. } \\
\text { \& Hook. f. ex S. } \\
\text { Moore }\end{array}$ & Craibeira & $\begin{array}{l}\mathrm{Cb}, \mathrm{Ct}, \mathrm{Fr} \\
\mathrm{Me}, \mathrm{Or}, \mathrm{Ot} \\
\mathrm{Tc},\end{array}$ & $\begin{array}{c}\mathrm{Ca}, \mathrm{Fl}, \mathrm{Fr}, \mathrm{Fo} \\
\mathrm{Ma}, \mathrm{Pc}\end{array}$ & 5,88 & 3 & 2,61 & 0,15 & 2,14 & 0,32 & 0,43 \\
\hline $\begin{array}{c}\text { Tabebuia } \\
\text { impetiginosa (Mart. } \\
\text { ex DC.) Standl }\end{array}$ & Pau d'arco roxo & $\begin{array}{c}\mathrm{Cb}, \mathrm{Ct}, \mathrm{Me} \\
\mathrm{Tc}\end{array}$ & $\mathrm{Ca}, \mathrm{Ma}$ & 0,18 & - & - & - & - & - & - \\
\hline Tabebuia sp. & Pau d'arco & $\begin{array}{l}\mathrm{Cb}, \mathrm{Ct}, \mathrm{Fr} \\
\mathrm{Me}, \mathrm{Or}, \mathrm{Tc}\end{array}$ & $\begin{array}{l}\mathrm{Ca}, \mathrm{Fl}, \mathrm{Fo} \\
\mathrm{Ma}, \mathrm{Pc}\end{array}$ & 0,6 & 13 & 3,55 & 0,65 & 1,72 & 1,18 & 0,35 \\
\hline $\begin{array}{c}\text { Boraginaceae } \\
\text { Cordia trichotoma } \\
\text { (Vell.) } \\
\text { Arráb. ex Steud. }\end{array}$ & Frei Jorge & $\mathrm{Cb}, \mathrm{Ct}, \mathrm{Tc}$ & $\mathrm{Ma}$ & 0,92 & - & - & - & - & - & - \\
\hline $\begin{array}{c}\text { Burseraceae } \\
\text { Commiphora } \\
\text { leptophloeos (Mart.) } \\
\text { J. B. Gillet } \\
\text { Capparaceae }\end{array}$ & Imburana & $\begin{array}{l}\mathrm{Cb}, \mathrm{Ct}, \mathrm{Fr} \\
\mathrm{Me}, \mathrm{Ot}, \mathrm{Tc} \\
\mathrm{Vt}\end{array}$ & $\begin{array}{l}\mathrm{Ca}, \mathrm{Fo}, \mathrm{La} \\
\mathrm{Ma}, \mathrm{Pc}\end{array}$ & 1,71 & 2 & 1,53 & 0,1 & 1,22 & 0,22 & 0,25 \\
\hline $\begin{array}{l}\text { Capparis jacobinae } \\
\text { Moric. ex Eichler }\end{array}$ & Icó & $\mathrm{Al}$ & Fr & 0,01 & - & - & - & - & - & - \\
\hline $\begin{array}{l}\text { Cynophalla flexuosa } \\
\text { (L.) J. Prese }\end{array}$ & $\begin{array}{c}\text { Feijão brabo/Feijão } \\
\text { de boi }\end{array}$ & $\mathrm{Cb}, \mathrm{Fr}, \mathrm{Tc}, \mathrm{Vt}$ & $\mathrm{Ca}, \mathrm{Fo}, \mathrm{Ma}$ & 0,09 & 3 & 0,7 & 0,15 & 0,23 & 0,32 & 0,05 \\
\hline
\end{tabular}




\begin{tabular}{|c|c|c|c|c|c|c|c|c|c|c|}
\hline Família / Espécie & Nome vernacular & Usos & $\begin{array}{l}\text { Partes das } \\
\text { plantas }\end{array}$ & $\mathbf{V U}$ & $\begin{array}{l}N^{\circ} \\
\text { Ind }\end{array}$ & VI & DeR & DoR & FRt & $\begin{array}{l}\text { Área } \\
\text { basal }\end{array}$ \\
\hline \multicolumn{11}{|l|}{ Celastraceae } \\
\hline $\begin{array}{c}\text { Maytenus rigida } \\
\text { Mart. }\end{array}$ & Bom nome & $\begin{array}{l}\mathrm{Cb}, \mathrm{Fr}, \mathrm{Me} \\
\mathrm{Tc}, \mathrm{Vt}\end{array}$ & $\mathrm{Ca}, \mathrm{Fo}, \mathrm{Ma}$ & 0,31 & - & - & - & - & - & - \\
\hline \multicolumn{11}{|l|}{ Choclospermaceae } \\
\hline $\begin{array}{c}\text { Choclospermum } \\
\text { insigne }\end{array}$ & Algodão brabo & Or & Pc & 0,01 & - & - & - & - & - & - \\
\hline $\begin{array}{c}\text { Combretaceae } \\
\text { Combretum } \\
\text { fruticosum (Coefl) } \\
\text { Stunts }\end{array}$ & Mufumbo & $\begin{array}{l}\mathrm{Cb}, \mathrm{Ct}, \mathrm{Me} \\
\mathrm{Ot}, \mathrm{Tc}\end{array}$ & $\mathrm{Ca}, \mathrm{Ma}, \mathrm{Pc}$ & 0,3 & - & - & - & - & - & - \\
\hline \multicolumn{11}{|l|}{ Euphorbiaceae } \\
\hline $\begin{array}{c}\text { Cnidoscolus } \\
\text { quercifolius Pohl }\end{array}$ & Favela & $\begin{array}{l}\mathrm{Cb}, \mathrm{Ct}, \mathrm{Fr} \\
\mathrm{Me}, \mathrm{Ot}, \mathrm{Tc} \\
\text { Va, Vt }\end{array}$ & $\begin{array}{l}\mathrm{Ca}, \mathrm{Fo}, \mathrm{Fr} \\
\mathrm{La}, \mathrm{Ma}, \mathrm{Tp}\end{array}$ & 0,49 & 1 & 0,2 & 0,05 & 0,04 & 0,11 & 0,01 \\
\hline $\begin{array}{c}\text { Croton } \\
\text { blanchetianus Baill. }\end{array}$ & Marmeleiro & $\begin{array}{l}\mathrm{Cb}, \mathrm{Ct}, \mathrm{Fr} \\
\mathrm{Me}, \mathrm{Ot}, \mathrm{Tc}\end{array}$ & $\begin{array}{l}\text { Ca, Fl, Fo, Fr, } \\
\text { Ma, Tp, Ra, } \\
\text { Se }\end{array}$ & 2,48 & 1400 & 159 & 70 & 38,8 & 49.84 & 7,88 \\
\hline $\begin{array}{c}\text { Croton rhamnifolius } \\
\text { Kunth. }\end{array}$ & Velame & $\mathrm{Fr}, \mathrm{Me}$ & $\mathrm{Ca}, \mathrm{Fo}, \mathrm{Ra}$ & 0,07 & 2 & 0,32 & 0,1 & 0,01 & 0,22 & 0 \\
\hline $\begin{array}{l}\text { Jatropha mollissima } \\
\text { (Pohl) Baill. }\end{array}$ & Pinhão brabo & $\begin{array}{l}\mathrm{Cb}, \mathrm{Fr}, \mathrm{Mr} \\
\mathrm{Me}, \mathrm{Ot}, \mathrm{Tc} \\
\mathrm{Vt}\end{array}$ & $\begin{array}{l}\text { Fo, La, Ma, } \\
\text { Se, Pc }\end{array}$ & 0,84 & 94 & 12,5 & 4,7 & 1,55 & 6,24 & 0,32 \\
\hline $\begin{array}{l}\text { Jatropha ribifolia } \\
\text { (Pohl) Baill. }\end{array}$ & Pinhão manso & $\mathrm{Mr}, \mathrm{Me}$ & Fo, Se & 0,03 & - & - & - & - & - & - \\
\hline $\begin{array}{c}\text { Manihot } \text { cf. } \\
\text { dichotoma Ule } \\
\text { Fabaceae }\end{array}$ & Maniçoba & $\mathrm{Al}, \mathrm{Fr}, \mathrm{Tc}, \mathrm{Va}$ & Fo, Ma, Tb & 0,26 & 11 & 2,73 & 0,55 & 1,11 & 1,08 & 0,23 \\
\hline $\begin{array}{l}\text { Amburana cearensis } \\
\text { (Allemão) A.C.Sm. }\end{array}$ & Cumarú & $\begin{array}{l}\mathrm{Cb}, \mathrm{Ct}, \mathrm{Fr} \\
\mathrm{Me}, \mathrm{Tc}\end{array}$ & $\begin{array}{l}\mathrm{Ca}, \mathrm{Fo}, \mathrm{Fr} \\
\mathrm{Ma}, \mathrm{Se}\end{array}$ & 0,44 & - & - & - & - & - & - \\
\hline $\begin{array}{c}\text { Anadenanthera } \\
\text { colubrina (Vell.) } \\
\text { Brenan }\end{array}$ & Angico & $\begin{array}{l}\mathrm{Cb}, \mathrm{Ct}, \mathrm{Fr} \\
\mathrm{Mr}, \mathrm{Me}, \mathrm{Ot}, \\
\mathrm{Tc}, \mathrm{Va}, \mathrm{Vt}\end{array}$ & $\begin{array}{l}\mathrm{Ca}, \mathrm{Ec}, \mathrm{Fo} \\
\mathrm{Fr}, \mathrm{Ma}, \mathrm{Pc}\end{array}$ & 2,86 & 36 & 14,6 & 1,8 & 9,81 & 3,01 & 1,99 \\
\hline $\begin{array}{l}\text { Bauhinia cheilantha } \\
\text { (Bong.) Steud. }\end{array}$ & Mororó & $\begin{array}{l}\mathrm{Cb}, \mathrm{Ct}, \mathrm{Fr} \\
\mathrm{Me}, \mathrm{Tc}\end{array}$ & $\mathrm{Ca}, \mathrm{Fo}, \mathrm{Ma}$ & 0,4 & 9 & 2,26 & 0,45 & 0,84 & 0,97 & 0,17 \\
\hline $\begin{array}{c}\text { Erythrina velutina } \\
\text { Willd. }\end{array}$ & Mulungú & $\begin{array}{l}\mathrm{Ct}, \mathrm{Me}, \mathrm{Or} \\
\mathrm{Ot}, \mathrm{Tc}, \mathrm{Va}\end{array}$ & $\begin{array}{l}\mathrm{Ca}, \mathrm{Ec}, \mathrm{Ma} \\
\mathrm{Pc}\end{array}$ & 0,3 & - & - & - & - & - & - \\
\hline $\begin{array}{c}\text { Hymenoca courbaril } \\
\text { L. }\end{array}$ & Jatobá & $\begin{array}{l}\mathrm{Al}, \mathrm{Cb}, \mathrm{Ct} \\
\mathrm{Me}, \mathrm{Tc}\end{array}$ & $\mathrm{Ca}, \mathrm{Fr}, \mathrm{Ma}$ & 0,22 & - & - & - & - & - & - \\
\hline Inga sp. & Ingazeira & $\begin{array}{l}\mathrm{Cb}, \mathrm{Ct}, \mathrm{Fr} \\
\mathrm{Me}, \mathrm{Ot}\end{array}$ & $\begin{array}{c}\mathrm{Ca}, \mathrm{Fo}, \mathrm{Ma} \\
\mathrm{Pc}\end{array}$ & 0,14 & - & - & - & - & - & - \\
\hline $\begin{array}{l}\text { Libidibia ferrea } \\
\text { (Mart. ex Tul.) } \\
\text { L.P.Queiroz }\end{array}$ & Jucá & $\begin{array}{l}\mathrm{Cb}, \mathrm{Ct}, \mathrm{Fr}, \\
\mathrm{Me}, \mathrm{Ot}, \mathrm{Tc}, \\
\mathrm{Me}\end{array}$ & $\begin{array}{l}\mathrm{Ca}, \mathrm{Fo}, \mathrm{Fr}, \\
\mathrm{Ma}\end{array}$ & 1,01 & 1 & 0,65 & 0,05 & 0,49 & 0,11 & 0,1 \\
\hline $\begin{array}{l}\text { Mimosa tenuiflora } \\
\text { (Willd.) Poir. }\end{array}$ & Jurema preta & $\begin{array}{l}\mathrm{Cb}, \mathrm{Ct}, \mathrm{Fr} \\
\mathrm{Me}, \mathrm{Ot}, \mathrm{Tc} \\
\text { Va, } \mathrm{Vt}\end{array}$ & $\begin{array}{l}\mathrm{Ca}, \mathrm{Ec}, \mathrm{Fl}, \\
\mathrm{Fo}, \mathrm{Fr}, \mathrm{Ma} \\
\mathrm{Pc}, \mathrm{Se}\end{array}$ & 2,4 & 14 & 15 & 0,7 & 13 & 1,29 & 2,65 \\
\hline $\begin{array}{c}\text { Piptadenia } \\
\text { stipulaceae (Benth.) } \\
\text { Ducke }\end{array}$ & Amorosa & $\mathrm{Me}$ & $\mathrm{Se}$ & 0,01 & - & - & - & - & - & - \\
\hline
\end{tabular}




\begin{tabular}{|c|c|c|c|c|c|c|c|c|c|c|}
\hline Família / Espécie & Nome vernacular & Usos & $\begin{array}{l}\text { Partes das } \\
\text { plantas }\end{array}$ & $\mathbf{V U}$ & $\begin{array}{l}N^{\circ} \\
\text { Ind }\end{array}$ & VI & DeR & DoR & FRt & $\begin{array}{l}\text { Área } \\
\text { basal }\end{array}$ \\
\hline $\begin{array}{c}\text { Poincianella } \\
\text { pyramidalis Tul. }\end{array}$ & Catingueira & $\begin{array}{l}\mathrm{Cb}, \mathrm{Ct}, \mathrm{Fr} \\
\mathrm{Mr}, \mathrm{Me}, \mathrm{Or} \\
\mathrm{Ot}, \mathrm{Tc}, \mathrm{Vt}\end{array}$ & $\begin{array}{c}\text { Ca, Ec, Fl, } \\
\text { Fo, Fr, Ma, Pc }\end{array}$ & 2,99 & 198 & 41,7 & 9,9 & 15 & 16,8 & 3,05 \\
\hline $\begin{array}{c}\text { Senna martiana } \\
\text { (Benth.) H.S. Irwin } \\
\text { Barneby } \\
\text { Malvaceae }\end{array}$ & Canafístula & $\mathrm{Cb}, \mathrm{Or}, \mathrm{Ot}, \mathrm{Tc}$ & $\mathrm{Ma}, \mathrm{Pc}$ & 0,04 & 5 & 2,86 & 0,25 & 2,29 & 0,32 & 0,47 \\
\hline $\begin{array}{l}\text { Chorisia glaziovii } \\
\text { (Kuntze) E. Santos }\end{array}$ & Barriguda & Or, Ot & $\mathrm{Pc}, \mathrm{Fr}$ & 0,03 & - & - & - & - & - & - \\
\hline $\begin{array}{c}\text { Pseudobombax } \\
\text { marginatum (A.St.- } \\
\text { Hil.,Juss.\& } \\
\text { Cambess.) A. } \\
\text { Robyns }\end{array}$ & Imbiratã & $\mathrm{Ct}, \mathrm{Me}, \mathrm{Ot}, \mathrm{Vt}$ & $\mathrm{Ca}, \mathrm{Fr}, \mathrm{Ma}$ & 0,13 & 1 & 0,23 & 0,05 & 0,07 & 0,11 & 0,01 \\
\hline $\begin{array}{c}\text { Meliaceae } \\
\text { Cedrela odorata L. } \\
\text { Myrtaceae }\end{array}$ & Cedro & $\mathrm{Me}$ & Fo & 0,01 & - & - & - & - & - & - \\
\hline $\begin{array}{c}\text { Eugenia uvalha } \\
\text { Cambess. } \\
\text { Olacaceae }\end{array}$ & Ubaia & $\mathrm{Al}, \mathrm{Fr}$ & Fr & 0,13 & - & - & - & - & - & - \\
\hline $\begin{array}{c}\text { Ximenia americana } \\
\text { L. }\end{array}$ & Ameixa & $\begin{array}{l}\mathrm{Cb}, \mathrm{Ct}, \mathrm{Fr} \\
\mathrm{Me}, \mathrm{Ot}, \mathrm{Tc} \\
\mathrm{Vt}\end{array}$ & $\begin{array}{l}\text { Ca, Ec, Fo, } \\
\text { Fr, Ma, Pc }\end{array}$ & 1 & - & - & - & - & - & - \\
\hline $\begin{array}{c}\text { Polygonaceae } \\
\text { Triplaris } \\
\text { gardneriana } \text { Wedd. } \\
\text { Rhamnaceae }\end{array}$ & Cuaçú & $\mathrm{Cb}, \mathrm{Fr}, \mathrm{Tc}$ & Fo, Ma & 0,05 & - & - & - & - & - & - \\
\hline $\begin{array}{l}\text { Ziziphus joazeiro } \\
\text { Mart. }\end{array}$ & Juazeiro & $\begin{array}{c}\mathrm{Al}, \mathrm{Cb}, \mathrm{Ct}, \mathrm{Fr}, \\
\mathrm{Me}, \mathrm{Or}, \mathrm{Ot} \\
\mathrm{Tc}\end{array}$ & $\begin{array}{l}\mathrm{Ca}, \mathrm{Ec}, \mathrm{Fo}, \\
\text { Fr, Ma, Pc }\end{array}$ & 3,02 & 2 & 0,79 & 0,1 & 0,47 & 0,22 & 0,1 \\
\hline $\begin{array}{c}\text { Rubiaceae } \\
\text { Tocoyena formosa }\end{array}$ & Jenipapo brabo & $\begin{array}{c}\mathrm{Cb}, \mathrm{Me}, \mathrm{Tc} \\
\mathrm{Vt}\end{array}$ & $\mathrm{Ca}, \mathrm{Ma}$ & 0,07 & 2 & 0,37 & 0,1 & 0,06 & 0,22 & 0,01 \\
\hline $\begin{array}{c}\text { Sapotaceae } \\
\text { Sideroxylon } \\
\text { obtusifolium (Roem } \\
\text { \& Schult.) T. D. } \\
\text { Penn. } \\
\text { Indetermidas }\end{array}$ & Quixabeira & $\begin{array}{c}\mathrm{Al}, \mathrm{Cb}, \mathrm{Ct}, \mathrm{Fr}, \\
\mathrm{Me}, \mathrm{Ot}, \mathrm{Tc}, \\
\mathrm{Vt}\end{array}$ & $\begin{array}{l}\mathrm{Ca}, \mathrm{Ec}, \mathrm{Fo} \\
\text { Fr, Ma, Pc }\end{array}$ & 3,71 & - & - & - & - & - & - \\
\hline Indet. 1 & Canela de ema & Fr, Tc & Fo, Ma & 0,02 & - & - & - & - & - & - \\
\hline Indet. 2 & Catinga branca & $\mathrm{Cb}, \mathrm{Ct}, \mathrm{Fr}, \mathrm{Me}$ & $\begin{array}{c}\mathrm{Ca}, \mathrm{Fo}, \mathrm{Ma} \\
\mathrm{Pc}\end{array}$ & 0,45 & - & - & - & - & - & - \\
\hline Indet. 3 & Guaxumba & $\mathrm{Ct}, \mathrm{Tc}$ & $\mathrm{Ma}$ & 0,11 & - & - & - & - & - & - \\
\hline Indet. 4 & Jaramataia & $\mathrm{Me}$ & Fo & 0,02 & - & - & - & - & - & - \\
\hline Indet. 5 & Jurema branca & $\begin{array}{l}\mathrm{Cb}, \mathrm{Ct}, \mathrm{Fr} \\
\mathrm{Mr}, \mathrm{Me}, \mathrm{Tc}\end{array}$ & $\begin{array}{c}\mathrm{Ca}, \mathrm{Fo}, \mathrm{Fr} \\
\mathrm{Ma}\end{array}$ & 0,44 & - & - & - & - & - & - \\
\hline Indet. 6 & Jurema de imbira & $\begin{array}{c}\mathrm{Cb}, \mathrm{Ct}, \mathrm{Fr}, \mathrm{Ot}, \\
\mathrm{Tc}\end{array}$ & $\mathrm{Fl}, \mathrm{Fo}, \mathrm{Fr}, \mathrm{Ma}$ & 0,56 & - & - & - & - & - & - \\
\hline Indet. 7 & Jureminha & $\mathrm{Va}$ & $\mathrm{Fl}$ & 0,01 & - & - & - & - & - & - \\
\hline Indet. 8 & Louro & $\mathrm{Ct}, \mathrm{Tc}$ & $\mathrm{Ma}$ & 0,14 & - & - & - & - & - & - \\
\hline
\end{tabular}




\begin{tabular}{|c|c|c|c|c|c|c|c|c|c|c|}
\hline Família / Espécie & Nome vernacular & Usos & $\begin{array}{c}\text { Partes das } \\
\text { plantas }\end{array}$ & $\mathbf{V U}$ & $\begin{array}{l}\mathrm{N}^{\circ} \\
\text { Ind }\end{array}$ & VI & DeR & DoR & FRt & $\begin{array}{l}\text { Área } \\
\text { basal }\end{array}$ \\
\hline Indet. 9 & Pau de serrote & $\begin{array}{c}\mathrm{Cb}, \mathrm{Ct}, \mathrm{Fr}, \mathrm{Ot}, \\
\mathrm{Tc}\end{array}$ & Fo, Ma, Pc & 0,1 & - & - & - & - & - & - \\
\hline Indet. 10 & Pau leite & $\mathrm{Me}$ & $\mathrm{Ca}$ & 0,03 & - & - & - & - & - & - \\
\hline Indet. 11 & Pau piranha & $\mathrm{Me}, \mathrm{Tc}, \mathrm{Vt}$ & $\mathrm{Ca}, \mathrm{Ma}$ & 0,1 & - & - & - & - & - & - \\
\hline Indet. 12 & Pau preto & $\mathrm{Ct}$ & $\mathrm{Ma}$ & 0,01 & - & - & - & - & - & - \\
\hline Indet. 13 & Quebra faca & $\mathrm{Cb}, \mathrm{Ct}$ & $\mathrm{Ma}$ & 0,02 & - & - & - & - & - & - \\
\hline Indet. 14 & Rabo de cavalo & $\mathrm{Ct}, \mathrm{Ot}, \mathrm{Tc}$ & $\mathrm{Ma}, \mathrm{Fo}$ & 0,21 & - & - & - & - & - & - \\
\hline
\end{tabular}

$\mathrm{Al}=$ Alimento $\mathrm{Cb}=$ Combustível; $\mathrm{Ct}=$ Construção $\mathrm{Fr}=$ Forragem; $\mathrm{Me}=$ Medicinal; $\mathrm{Ot}=$ Outros; $\mathrm{Or}=$ Ornamental; $\mathrm{Tc}=$ Tecnologia; $\mathrm{Va}=$ Veneno-Abortivo; $\mathrm{Vt}=$ Veterinário; $\mathrm{Mr}=$ Mágico-religioso. $\mathrm{Ca}=\mathrm{Casca} ; \mathrm{Ec}=$ Entrecasca; Fo $=$ Folha; Fl = Flor; Fr $=$ Fruto; La $=$ Látex; $\mathrm{Ma}=$ Madeira Pc $=$ Planta Completa; Ra $=$ Raiz; $\mathrm{Se}=$ Semente; $\mathrm{Ce}=\mathrm{Cera} ; \mathrm{Tb}=$ Tubérculo. $\mathrm{VU}=$ Valor de uso; $\mathrm{N}^{\circ} \mathrm{IND}=$ Número de indivíduos; $\mathrm{VI}=$ valor de importância; DeR = Densidade relativa; DoR = Dominância relativa; $F R=$ Frequência relativa.

A quantidade de vezes que uma planta é citada está relacionada à sua importância cultural (Rosero-Toro, Romero-Duque, Santos-Fita, \& Ruan-Soto, 2018). O elenco de espécies mais citadas para este estudo é semelhante ao encontrado por outros pesquisadores anteriormente em regiões circunvizinhas (Guerra et al., 2012; Leite et al., 2012; Soares et al., 2013). Entretanto, nem sempre a importância cultural irá representar a realidade de extração de uma espécie, para isto, a aplicação de índices e metodologias auxiliares deve ser utilizada.

Neste estudo, as espécies mencionadas pelos informantes foram organizadas em 11 categorias de uso, sendo elas: alimento, combustível, construção, forragem, medicinal, "outros", categoria que agrupa tipos de uso com baixo número de citações como sombra e bioindicação; ornamental, tecnologia, veneno-abortivo, veterinário e mágico-religioso. As espécies mais citadas se enquadram em no mínimo 7 categorias de uso, demonstrando possuir grande representatividade e diversidade utilitária (ver Tabela 1).

Em relação ao valor de uso (VU) as espécies de maior destaque foram $T$. aurea (VU $=5,88)$, Aspidosperma pyrifolium Mart. $(3,74)$ e S. obtusifolium $(3,71)$. Em estudo semelhante, em um trecho de mata ciliar no semiárido do estado do Pernambuco, também foi encontrado o maior valor de uso para T. aurea (Ferraz et al., 2006). Os pesquisadores, da mesma forma que no presente estudo, utilizaram o valor de uso como métrica para validar a importância utilitária das espécies.

Em estudo realizado na região do Sertão do estado da Paraíba, $T$. aurea também foi identificada como espécie com maior valor de uso (Guerra et al., 2012). Entretanto, os autores também utilizaram um cálculo diferenciado para esse índice, usando literatura específica como base (Albuquerque \& Lucena, 2005; Lucena, Albuquerque, et al., 2007; Lucena, Araújo, \& Albuquerque, 2007; Lucena et al., 2012), onde as citações de uso são organizadas em suas seções, usos contemporâneos ou atual, e uso reconhecido pela comunidade, mas não efetivo, chamado de uso potencial. Com essa separação, os autores conseguem diferenciar os valores de uso de espécies com usos potencial e atual.

O objetivo desta pesquisa não inclui o cálculo diferenciado do índice valor de uso, como exemplificado acima, entretanto, recomenda-se para estudos futuros, e semelhantes a este, que possam utilizar essa distinção para auxiliar em discussões sobre a dinâmica do uso e conhecimento tradicional acerca das espécies vegetais, o que pode trazer importantes contribuições para a conservação dos recursos naturais. 
A exemplo, no estado da Bahia, em estudo etnobotânico e populacional de $M$. urundeuva, foi concluído que mais de $70 \%$ dos informantes faziam o uso atual da espécie (Barros, Nascimento, $\&$ Medeiros, 2016), dado gerado a partir de índice diferenciado de valor de uso (Lucena et al., 2012). No caso do presente estudo, $M$. urundeuva mesmo sendo a mais citada pelos residentes da comunidade Santa Rita, não está entre as espécies que possuem maior valor de uso. Tal fato também foi observado em outro estudo no semiárido paraibano (Soares et al., 2013).

As espécies enquadradas em uma maior diversidade de categorias foram Schinopsis brasiliensis Engl., Aspidosperma pyrifolium Mart., Anadenanthera colubrina (Vell.) Brenan, Poincianella pyramidalis Tul, (todas citadas em 9 categorias). As espécies que apresentaram maior número de partes úteis foram C. blanchetianus e M. tenuiflora (ambas com 8 partes úteis) (Tabela 1).

Poucas espécies apresentaram usos múltiplos. Ao classificar as espécies levando em consideração a diversidade de usos, foi observado que apenas duas apresentaram mais de 45 usos, e somente três apresentaram de 40 a 45 usos, enquanto 23 espécies apresentaram de 1 a 5 usos (fig. 2). As partes mais utilizadas foram a madeira (2624 citações), casca (731 citações) e folha (384 citações) (fig. 2).

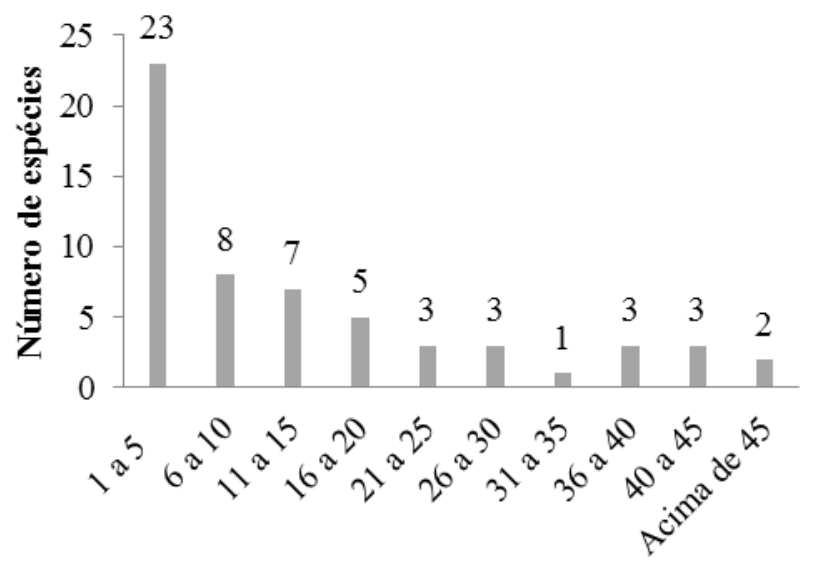

Número de usos por espécie

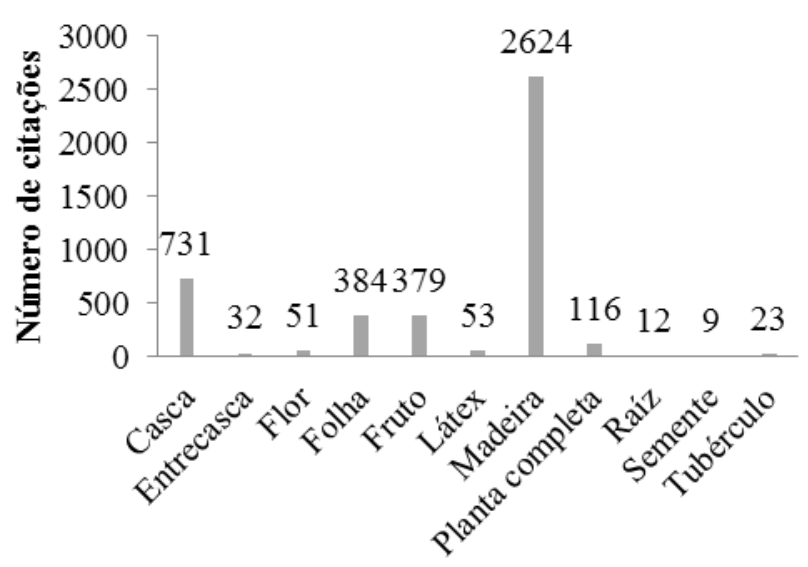

Parte Usada

Fig. 2. Número de espécies e distribuição em classes conforme os usos atribuídos e número de citações por partes das plantas utilizadas pelos moradores da comunidade Santa Rita, município do Congo, estado da Paraíba, Nordeste do Brasil.

A relação entre as categorias de uso e as partes de plantas mais utilizadas também foram encontradas em demais estudos semelhantes (Guerra et al., 2012; Leite et al., 2012; N. Silva et al., 2014; Soares et al., 2013). O significado cultural das categorias construção e tecnologia justificam a quantidade de citações para uso da madeira. É alertado para a preferência por parte da coleta de madeira verde, de acordo com a finalidade do uso, e que isto implica na retirada de todo o tronco do indivíduo, restando tocos baixos que dificultam o processo de regeneração (Barros et al., 2016). Além do que, ao fazer este tipo de corte, concluíram que os informantes escolhiam os indivíduos pelo diâmetro do tronco, quanto maior o diâmetro, mais procuradas para extração. 
Tabela 2. Número de espécies e número de citações das plantas úteis por categoria na comunidade Santa Rita, município do Congo, estado da Paraíba, Nordeste do Brasil.

\begin{tabular}{lcr}
\hline Categoria de uso & Número de espécies & $\begin{array}{c}\text { Número de citações } \\
(\%)\end{array}$ \\
\hline Alimento & 8 & $240(5,3 \%)$ \\
Combustível & 34 & $690(15,1 \%)$ \\
Construção & 34 & $1035(22,6 \%)$ \\
Forragem & 33 & $443(9,7 \%)$ \\
Mágico-religioso & 5 & $8(0,2 \%)$ \\
Medicinal & 37 & $730(16 \%)$ \\
Ornamental & 11 & $15(0,3 \%)$ \\
Outros & 26 & $196(4,3 \%)$ \\
Tecnologia & 42 & $1027(22,5 \%)$ \\
Veneno-abortivo & 8 & $100(2,2 \%)$ \\
Veterinário & 18 & $87(1,9 \%)$ \\
\hline
\end{tabular}

O número de citações de casca como parte utilizada é referente a categoria medicinal. Este tipo de resultado é geralmente encontrado em ambientes decíduos, caso das áreas de Caatinga e pode ser relacionado a hipótese da sazonalidade climática (Albuquerque, 2006; Medeiros, Haydée Ladio, \& Albuquerque, 2013). Em um estudo comparativo sobre conhecimento botânico local sobre plantas medicinais em sete comunidades rurais do estado da Paraíba, foi encontrado o uso predominante da casca para todas as áreas de estudo, apesar de que as principais espécies utilizadas (M. urundeuva, T. aurea e S. obtusifolium), também possuem grande quantidade de princípios ativos nas folhas (Marreiros et al., 2015).

\section{Inventário de vegetação}

Das 56 espécies vegetais citadas nas entrevistas, o levantamento fitossociológico registrou 24, pertencentes a 21 gêneros e 12 famílias. Dos 2000 indivíduos amostrados, 55 não foram identificados.

As famílias de maior destaque em relação ao número de indivíduos foram Euphorbiaceae (1524 indivíduos), Fabaceae (271 indivíduos) e Apocynaceae (117 indivíduos). Entre as espécies, houve destaque para Croton blanchetianus Baill (marmeleiro), com 1400 indivíduos, seguido por Poincianella pyramidalis Tul. (catingueira) (198 indivíduos) e Aspidosperma pyrifolium Mart. (pereiro) (117 indivíduos) (Tabela 1).

Um estudo sobre a presença e uso de espécies da família Euphorbiaceae na Chapada do Araripe (Nordeste do Brasil) destaca a ampla distribuição e uso desta família, inferindo ainda uma maior riqueza de espécies do gênero Croton, especificamente Croton heliotropiifolius Kunth, semelhante ao "marmeleiro" e popularmente chamado de "velame" (Crepaldi, Campos, Albuquerque, \& Sales, 2016). Os autores mencionam ainda que as Euphorbiaceae se destacam nos países dos trópicos tanto pela riqueza, quanto pela sua utilização, já documentada, em comunidades rurais, o que pode ser verificado pela diversidade de espécies e usos nessa família em estudos realizados em Serra Leoa (Kanteh \& Norman, 2015), Tanzânia (Amri \& Kisangau, 2012), Índia (Ravikumar et al., 2011) e África do Sul (Philander, 2011).

A maior disponibilidade desses indivíduos já era explicada em literaturas precedentes a este estudo (Lucena \& Alves, 2010; J. S. Silva, Sales, Gomes, \& Carneiro-Torres, 2010), que mencionam a família Euphorbiaceae como de característica pioneira, que é facilmente encontrada em ambientes ruderais e antropizados. Também ressaltam que espécies do gênero Croton são amplamente encontradas em diferentes biomas como Floresta Amazônica, Mata Atlântica e Caatinga. 
Tratando-se da Caatinga, diversos estudos apontam as espécies $C$. blanchetianus, $P$. pyramidalis, A. pyrifolium e Mimosa tenuiflora (Willd) (jurema preta) como as mais abundantes na composição dos estratos lenhosos amostrados em diferentes condições e regiões (Dario, 2017; Lacerda \& Barbosa, 2018; Lima \& Coelho, 2018; Luna, Andrade, \& Souto, 2018; Sabino, Cunha, \& Santana, 2016) corroborando com o elenco de espécies encontrado neste estudo.

Sobre a presente pesquisa, apesar de haver registro representativo de famílias e gêneros, o que poderia ser interpretado como indicador de maior diversidade, C. blanchetianus representa 70\% dos indivíduos inventariados, o que implica em baixa diversidade e alto nível de antropização local. Em estudo em um fragmento florestal no Ceará foi elucidado que a grande quantidade de indivíduos do gênero Croton, indicam áreas em provável estado de recuperação, por serem espécies pioneiras (Lima \& Coelho, 2018). O dados aqui apresentados, reforçam o contínuo e avançado estado geral de degradação da Caatinga, enquanto ecossistema (Silva et al. 2017)

Resultado semelhante foi encontrado ao testar a hipótese da aparência ecológica, em uma comunidade rural na região do Seridó do estado da Paraíba (Lucena et al., 2014). Os autores analisaram dados fitossociológicos de duas regiões distintas, separando em conservada e degradada (onde havia extração de recursos por parte da população local), e encontraram dominância de C. blanchetianus na localidade antropizada da amostra. Da mesma maneira, corroboram com esta mesma lógica os estudos realizados pelo mesmo grupo de pesquisadores em diferentes regiões do domínio Caatinga no decorrer de alguns anos (Carvalho et al., 2012; Guerra et al., 2015, 2012; Leite et al., 2012).

As espécies com maior valor de importância (VI) e frequência relativa (FRt) registrado foram C. blanchetianus $(\mathrm{VI}=158,60 ; \mathrm{FRt}=49,89), P$. pyramidalis $(\mathrm{VI}=41,68 ; \mathrm{FRt}=16,79)$ e $A$. pyrifolium $(\mathrm{VI}=19,28 ; \mathrm{FRt}=10,55)$. Em relação à dominância relativa $(\mathrm{DoR})$, se sobressaíram C. blanchetianus $(\mathrm{DoR}=38,76)$, P. pyramidalis $(14,99)$ e $M$. tenuiflora $(13,04)$ (Tabela 1$)$.

Os dados fitossociológicos apresentados neste estudo, não indicam apenas a degradação dos fragmentos de matas que ficam nas proximidades da comunidade rural de Santa Rita, mas possibilitam demonstrar a situação da disponibilidade de espécies, tornando possível a identificação de disfunções estruturais nas populações ecológicas (Barros et al., 2016).

Os mesmos autores (Barros et al., 2016), ao tratar especificamente da distribuição e uso de Myracrodruon urundeuva Allemão, argumentam que o contexto socioeconômico das comunidades rurais do semiárido influencia na retirada de recursos madeireiros nas matas locais, prejudicando diretamente espécies versáteis (com muitas formas de uso) e os seus indivíduos de maior diâmetro. Propõe, portanto, que os pesquisadores de acordo com os seus objetos de estudo, se atentem para a criação de gerenciamento comunitário, onde se possa fazer uma rotatividade das áreas de extração, permitindo a regeneração e recuperação de zonas com maior nível de degradação local.

Ressaltando que o presente estudo visa analisar a disponibilidade de espécies vegetais admitidas como úteis pelas populações locais, se recomenda que a interpretação dos dados seja realizada mediante a relevância da importância cultural e as formas de uso das espécies vegetais (Barros et al., 2016). Desta forma, é possível inferir melhores conclusões que sirvam como subsídio teórico para planos e medidas para a conservação de espécies de importância econômica, para populações locais, e o equilíbrio das populações ecológicas (Ali, Perveen, \& Qaiser, 2015).

Em pesquisa realizada em território indígena Fulni-ô, no estado do Pernambuco pesquisadores utilizaram um outro índice (IPC - Índice de Prioridade de Conservação) que engloba dimensões biológicas e culturais, e utiliza dados sobre área de extração, método e frequência de coleta e importância local, para determinar prioridades de conservação local de plantas (Dzerefos \& Witkowski, 2001) Concluíram que o IPC não foi eficiente para a previsão de mudanças 
relacionando conhecimento tradicional, prioridade de conservação de espécies e o desaparecimento/disponibilidade de espécies raras na vegetação local (Souza et al., 2017).

No presente estudo, duas das três espécies com maior valor de uso (T. aurea e S. obtusifolium), são espécies características de estágios sucessionais mais avançados, enquanto que as espécies mais disponíveis na vegetação são todas pioneiras $(C$. blanchetianus, $P$. pyramidalis e $A$. pyrifolium), isto implica na condução de uma investigação mais específica sobre a maneira como as populações estão utilizando as espécies vegetais, a forma e frequência de extração; para verificar se a característica degradada dos fragmentos inventariados de mata é reflexo da tendência de maior procura de algumas espécies com maior área basal por parte dos habitantes locais, ou apenas reflete o alto grau generalizado de degradação do ecossistema caatinga.

Dentro do aspecto dinâmico do conhecimento tradicional, uma outra possibilidade é que as espécies mais utilizadas podem estar sendo extraídas, mas em novos locais de coleta; ou a degradação, e por fim, baixa disponibilidade das espécies com maior importância utilitária na vegetação local, pode resultar na substituição destas espécies por outras com maior disponibilidade e maior capacidade de regeneração.

\section{CONCLUSÃO}

Os dados apresentados corroboram com diversos pesquisadores e demonstram a relevância do estudo da estrutura populacional de espécies vegetais úteis, associando variáveis biológicas e culturais, a fim de compreender quais recursos florestais são mais afetados e os motivos culturais disto, podendo contribuir para a manutenção e sustentabilidade desse sistema.

Os parâmetros fitossociológicos, apoiados na literatura, indicam níveis de degradação local, entretanto, os dados se mostram limitados para que se possa inferir sobre o nível de impacto e a sustentabilidade dos recursos vegetais locais. A descrição do saber local se torna instrumento para a preservação dos recursos naturais e subsídio para planos de gestão participativa, priorizando a inclusão das populações tradicionais.

Este estudo indica que a composição da vegetação local na comunidade de Santa Rita é oriunda de altos níveis de antropização, sendo necessário maior detalhamento na dinâmica de uso e retirada dos indivíduos que demonstraram maior significado cultural e valor de uso.

\section{REFERÊNCIAS}

Albuquerque, U. P. (2006). Re-examining hypotheses concerning the use and knowledge of medicinal plants: A study in the Caatinga vegetation of NE Brazil. Journal of Ethnobiology and Ethnomedicine, 2. https://doi.org/10.1186/1746-4269-2-30

Albuquerque, U. P., \& Andrade, L. H. C. (2002). Conhecimento botânico tradicional e conservação em uma área de caatinga no estado de Pernambuco, Nordeste do Brasil. Acta Botanica Brasilica, 16(3), 273-285.

Albuquerque, U. P., \& Andrade, L. H. C. (2002). Uso de recursos vegetaisn da Caatinga: O caso do Agreste do estado de Pernambuco (Nordeste do Brasil). Interciencia, 27(7), 336-346. Retrieved from http://www.scielo.org.ve/scielo.php?script=sci_arttext\&pid=S0378-18442002000700002

Albuquerque, U. P., \& Lucena, R. F. P. (2005). Can apparency affect the use of plants by local people in tropical forests? Interciência, 30(8), 506-511. Retrieved from http://www.scielo.org.ve/scielo.php?script=sci_arttext\&pid=S0378-18442005000800013

Albuquerque, U. P., Lucena, R. F. P., \& Alencar, N. L. (2010). Métodos e técnicas para coleta de dados etnobiológicos. In U. P. Albuquerque, R. F. P. Lucena, \& L. V. F. C. Cunha (Eds.), Métodos e técnicas na pesquisa etnobiológica e etnoecológica (pp. 39-64). 
Recife: NUPEEA.

Albuquerque, U. P., Medeiros, P. M., \& Casas, A. (2015). Evolutionary Ethnobiology. In U. P. Albuquerque, P. M. Medeiros, \& A. Casas (Eds.), Evolutionary Ethnobiology (pp. 15). Switzerland: Springer.

Ali, S., Perveen, A., \& Qaiser, M. (2015). Vegetation structure, edaphalogy and ethnobotany of Mahaban and Malka (District Buner) KPK, Pakistan. Pakistan Journal of Botany, 47(SI), 15-22.

Amri, E., \& Kisangau, D. P. (2012). Ethnomedicinal study of plants used in villages around Kimboza forest reserve in Morogoro, Tanzania. Journal of Ethnobiology and Ethnomedicine, 8(1), 1. https://doi.org/10.1186/1746-4269-8-1

Araújo, E. L., \& Ferraz, E. M. N. (2010). Amostragem da vegetação nos estudos etnobotânicos. In U. P. Albuquerque, R. F. P. Lucena, \& L. V. F. C. Cunha (Eds.), Métodos e Técnicas na Pesquisa Etnobiológica e Etnoecológica (pp. 223-253). Recife: Núcleo Publicações em Ecologia e Etnobotânica Aplicada (NUPEEA).

Barros, F. N., Nascimento, V. T., \& Medeiros, P. M. (2016). Ethnobotany and Population Status of Myracrodruon urundeuva Allemão in Rural Northeastern Brazill. Economic Botany, 70(1), 79-84. https://doi.org/10.1007/s12231-015-9329-4

Carvalho, T. K. N., Sousa, R. F., Meneses, S. S. S., Ribeiro, J. P. O., Félix, L. P., \& Lucena, R. F. P. (2012). Plantas usadas por uma comunidade rural na Depressão Sertaneja no Nordeste do Brasil. Revista de Biologia e Farmácia, 4(Especial), 92-120.

Coutinho, P. C., Soares, Z. A., Ferreira, E. C., Souza, D. V., Oliveira, R. S., \& Lucena, R. F. P. (2015). Knowledge and use of medicinal plants in the Semiarid Region of Brazil. Brazilian Journal of Biological Sciences, 2(3), 51-74.

Crepaldi, C. G., Campos, J. L. A., Albuquerque, U. P., \& Sales, M. F. (2016). Richness and ethnobotany of the family Euphorbiaceae in a tropical semiarid landscape of Northeastern Brazil. South African Journal of Botany, 102, 157-165. https://doi.org/10.1016/j.sajb.2015.06.010

Dario, F. R. (2017). Estudo fitossociológico de uma área de caatinga em estágio inicial de sucessão ecológica no estado da Paraíba, Brasil. Geotemas, 7(1), 71-83.

Dzerefos, C. M., \& Witkowski, E. T. F. (2001). Density and potential utilization of medicinal grassland plants from Abe Bailey Nature Reserve, South Africa. Biodiversity and Conservation, 10, 1875-1896. https://doi.org/10.1023/A:1013177628331

Ferraz, J. S. F., Albuquerque, U. P., \& Meunier, I. M. J. (2006). Valor de uso e estrutura da vegetação lenhosa às margens do riacho do Navio, Floresta, PE, Brasil. Acta Bot. Bras., 20(1), 125-134. Retrieved from http://www.scielo.br/pdf/\%0D/abb/v20n1/12.pdf

Forzza, R. C., Baumgratz, J. F. A., Bicudo, C. E. M., Canhos, D. A. L., Carvalho, A. A., Coelho, M. A. N., ... Zappi, D. C. (2012). New Brazilian Floristic List Highlights Conservation Challenges. BioScience, 62(1), 39-45. https://doi.org/10.1525/bio.2012.62.1.8

Galeano, G. (2000). Forest Use at the Pacific Coast of Chocó, Colombia: A Quantitative Approach. Economic Botany, 54(3), 358-376.

Guerra, N. M., Carvalho, T. K. N., Ribeiro, J. E. S., Ribeiro, J. P. O., Barbosa, A. R., Lima, J. R. F., ... Lucena, R. F. P. (2015). Ecological Apparency Hypothesis and Plant Utility in the Semiarid Region of Brazil. Ethnobotany Research \& Applications, (December), 423-435.

Guerra, N. M., Leite, A. P., Souza, A. S., Ribeiro, J. E. S., Ribeiro, J. P. O., Oliveira, R. S., ... Lucena, R. F. P. (2014). Uso de algaroba (Prosopis juliflora (S .W.) DC ) en las comunidades tradicionales de las regiones semiáridas del Nordeste de Brasil. Gaia Scientia, Especial, 124-136.

Guerra, N. M., Ribeiro, J. E. S., Carvalho, T. K. N., Pedrosa, K. M., Félix, L. P., \& Lucena, R. F. P. (2012). Usos locais de espécies vegetais nativas em uma comunidade rural no Semiárido Nordestino (São Mamede, Paraíba, Brasil). Biofar, Especial, 184-209.

IBGE. Instituto Brasileiro de Geografia e Estatística. (2018). IBGE Cidades. Retrieved July 15, 2018, from https://cidades.ibge.gov.br/brasil/pb/congo/panorama

IPCC : Summary for Policymakers. (2013). Climate Change 2013: The Physical Science Basis. Contribution of Working Group I to the Fifth Assessment Report of the 
Intergovernmental Panel on Climate Change. (T. F. Stocker, D. Qin, G.-K. Plattner, M. M. B. Tignor, S. K. Allen, J. Boschung, ... P. M. Midgley, Eds.). Cambridge: Cambridge University Press. Retrieved from www.cambridge.org

Kanteh, S. M., \& Norman, J. E. (2015). Diversity of plants with pesticidal and medicinal properties in southern Sierra Leone. Biological Agriculture \& Horticulture, 31(1), 1827. https://doi.org/10.1080/01448765.2014.945621

Lacerda, A. V., \& Barbosa, F. M. (2018). Fitossociologia de Vegetação Arbustivo-Arbórea de uma Área Ribeirinha, Semiárido Paraibano, Brasil. Gaia Scientia, 12(2), 34-43. https://doi.org/10.22478/ufpb.1981-1268.2018v12n2.35719

Leite, A. P., Pedrosa, K. M., Lucena, C. M., Nunes, T. K. C., Félix, L. P., \& Lucena, R. F. P. (2012). Uso e conhecimento de espécies vegetais úteis em uma comunidade rural no Vale do Piancó (Paraíba, Nordeste, Brasil). Biofar, Especial, 133-157.

Lima, B. G., \& Coelho, M. D. F. B. (2018). Fitossociologia e estrutura de um fragmento florestal da caatinga, Ceará, Brasil. Ciência Florestal, 28(2), 809-819. https://doi.org/10.5902/1980509832095

Liporacci, H. S. N., Hanazaki, N., Ritter, M. R., \& Araújo, E. L. (2017). Where are the Brazilian ethnobotanical studies in the Atlantic Forest and Caatinga? Rodriguesia, 68(4), 1225-1240. https://doi.org/10.1590/2175-7860201768407

Lucena, C. M., Carvalho, T. K. N., Marín, E. A., Nunes, E. N., Oliveira, R. S., Melo, J. G., ... Lucena, R. F. P. (2014). Potencial medicinal de cactáceas en la región semiárida del Nordeste de Brasil. Gaia Scientia, Especial P, 36-50.

Lucena, C. M., Carvalho, T. K. N., Ribeiro, J. E. S., Quirino, Z. G. M., Casas, A., \& Lucena, R. F. P. (2015). Conhecimento botânico tradicional sobre cactáceas no semiárido do Brasil. Gaia Scientia, 9(2), 77-90. Retrieved from http://periodicos.ufpb.br/index.php/gaia/article/viewFile/24207/13285

Lucena, C. M., Lucena, R. F. P., Costa, G. M., Carvalho, T. K. N., Costa, G. G. da S., Alves, R. R. N., ... Nunes, E. N. (2013). Use and knowledge of Cactaceae in Northeastern Brazil. Journal of Ethnobiology and Ethnomedicine, 9(62). https://doi.org/10.1186/1746-4269-9-62

Lucena, M. F. A., \& Alves, M. (2010). Notas taxonômicas para Euphorbiaceae s.l. do Nordeste do Brasil. Hoehnea, 37(1), 71-85. Retrieved from http://www.scielo.br/pdf/hoehnea/v37n1/v37n1a05.pdf

Lucena, R. F. P., Albuquerque, U. P., Monteiro, J. M., Almeida, C. F. C. B. R., Florentino, A. T. N., \& Ferraz, J. S. F. (2007). Useful Plants of the Semi-Arid Northeastern Region of Brazil - A Look at their Conservation and Sustainable Use. Environmental Monitoring and Assessment, 125(1-3), 281-290. https://doi.org/10.1007/s10661-006-9521-1

Lucena, R. F. P., Araújo, E. L., \& Albuquerque, U. P. (2007). Does the local availability of woody Caatinga plants (Northeastern Brazil) explain their use value. Economic Botany, 61(4), 347-361. Retrieved from http://www.bioone.org/doi/abs/10.1663/00130001(2007)61\%5B347:DTLAOW\%5D2.0.CO\%3B2

Lucena, R. F. P., Medeiros, P. M., Araújo, E. L., Alves, A. G. C., \& Albuquerque, U. P. (2012). The ecological apparency hypothesis and the importance of useful plants in rural communities from Northeastern Brazil : An assessment based on use value. Journal of Environmental Management, 96, 106-115. https://doi.org/10.1016/j.jenvman.2011.09.001

Lucena, R. F. P., Pedrosa, K. M., Carvalho, T. K. N., Guerra, N. M., Ribeiro, J. E. S., \& Ferreira, E. C. (2017). Conhecimento local e uso de espécies vegetais nativas da região da Serra de Santa Catarina, Paraíba, Nordeste do Brasil. FLOVET, 1(9), 158-179.

Lucena, R. F. P., Sousa, R. F., Guerra, N. M., Ribeiro, J. E. S., Leite, A. P., Abreu, D. B. O., ... Nunes, E. N. (2014). The ecological apparency hypothesis and dry tropical forests: An ethnobotanical assessment. Etnoecológica, 10(9), 70-86.

Luna, R. G., Andrade, A. P., \& Souto, J. S. (2018). Análise florística e fitossociológica de quatro áreas de caatinga sob diferentes densidades de caprinos no Cariri Paraibano, Brasil. Revista Brasileira de Gestão Ambiental e Sustentabilidade, 5(9), 191-229. https://doi.org/10.21438/rbgas.050913 
Marreiros, N. A., Ferreira, E. C., Lucena, C. M., \& Lucena, R. F. P. (2015). Conhecimento botânico tradicional sobre plantas medicinais no semiárido da paraíba (Nordeste , Brasil). Revista Ouricuri, 5(1), 110-144.

Medeiros, P. M., Haydée Ladio, A., \& Albuquerque, U. P. (2013). Patterns of medicinal plant use by inhabitants of Brazilian urban and rural areas: A macroscale investigation based on available literature. Journal of Ethnopharmacology, 150, 729-746. https://doi.org/10.1016/j.jep.2013.09.026

Mendes, J. S., Chaves, L. H. G., \& Chaves, I. B. (2008a). Qualidade de água para consumo humano em comunidades rurais do município de Congo, PB. Revista Ciência Agronômica, 39(2), 333-342. $\quad$ Retrieved from http://ccarevista.ufc.br/seer/index.php/ccarevista/article/view/68

Mendes, J. S., Chaves, L. H. G., \& Chaves, I. B. (2008b). Variabilidade temporal da fertilidade, salinidade e sodicidade de solos irrigados no município de Congo, PB. Revista Brasileira de Ciências Agrárias, 3(1), 13-19. Retrieved from www.agraria.ufrpe.brProtocolo168-25/07/2007•Aprovadoem04/03/2008

Pedrosa, K. M., Almeida, H. A., Ramos, M. B., Barboza, R. R. D., \& Lopes, S. F. (2019). Local representation of change and conservation of a Brazilian Caatinga refuge. Biotemas, 32(3), 105-116. https://doi.org/10.5007/2175-7925.2019v32n3p105

Pedrosa, K. M., Lima, E. Q., Lucena, C. M., Carvalho, T. K. N., Ribeiro, J. E. S., Marín, E. A., ... Lucena, R. F. P. (2015). Local Botanical Knowledge about Sideroxylon Communities in the Semi- Arid Region of Brazil. Ethnobotany Research \& Applications, 14, 463-477.

Philander, L. A. (2011). An ethnobotany of Western Cape Rasta bush medicine. Journal of Ethnopharmacology, 138(2), 578-594. https://doi.org/10.1016/J.JEP.2011.10.004

Phillips, O., \& Gentry, A. H. (1993a). The useful plants of Tambopata, Peru: I. Statistical hypotheses tests with a new quantitative technique. Economic Botany, 47(1), 15-32. https://doi.org/10.1007/BF02862203

Phillips, O., \& Gentry, A. H. (1993b). The useful plants of Tambopata, Peru: II. Additional hypothesis testing in quantitative ethnobotany. Economic Botany, 47(1), 33-43. https://doi.org/10.1007/BF02862204

Ravikumar, S., Gokulakrishnan, R., Palaniselvan, G., Vinoth, R., Vijayakumar, R., \& UmaPandi, M. (2011). Ethnobotanical Survey of Coastal Medicinal Plants Along the Palk Strait Coast of South India. Journal of Herbs, Spices \& Medicinal Plants, 17(2), 69-84. https://doi.org/10.1080/10496475.2011.584823

Rosero-Toro, J. H., Romero-Duque, L. P., Santos-Fita, D., \& Ruan-Soto, F. (2018). Cultural significance of the flora of a tropical dry forest in the Doche vereda (Villavieja, Huila, Colombia). Journal of Ethnobiology and Ethnomedicine, 14(22). https://doi.org/10.1186/s13002-018-0220-0

Rossato, S. C., Leitão-Filho, H. F., \& Begossi, A. (1999). Ethnobotany of caiçaras of the Atlantic Forest coast (Brazil). Economic Botany, 53(4), 387-395. https://doi.org/10.1007/BF02866716

Sabino, F. G. S., Cunha, M. do C. L., \& Santana, G. M. (2016). Estrutura da Vegetação em Dois Fragmentos de Caatinga Antropizada na Paraíba. Floresta e Ambiente, 23(4), 487-497. https://doi.org/10.1590/2179-8087.017315

Santoro, F. R., Nascimento, A. L. B., Soldati, G. T., Ferreira Júnior, W. S., \& Albuquerque, U. P. (2018). Evolutionary ethnobiology and cultural evolution: opportunities for research and dialog. Journal of Ethnobiology and Ethnomedicine, 14(1), 1. https://doi.org/10.1186/s13002-017-0199-y

Silva, J. M. C., Leal, I. R., \& Tabarelli, M. (2017). Caatinga - The Largest Tropical Dry Forest Region in South America. Gewerbestrasse: Springer.

Silva, J. S., Sales, M. F., Gomes, A. P. S., \& Carneiro-Torres, D. S. (2010). Sinopse das espécies de Croton L. (Euphorbiaceae) no estado de Pernambuco, Brasil. Acta Botanica Brasilica, 24(2), 441-453. https://doi.org/10.1590/S010233062010000200015 
Recibido:

16/mayo/2019

Aceptado:

5/julio/2020
Silva, N., Lucena, R. F. P., Lima, J. R. F., Lima, G. D. S., Carvalho, T. K. N., Sousa Júnior, S. P., \& Alves, C. A. B. (2014). Conhecimento e Uso da Vegetação Nativa da Caatinga em uma Comunidade Rural da Paraíba , Nordeste do Brasil. Boletim Do Museu de Biologia Mello Leitão, 34, 5-37.

Soares, Z. A., Lucena, R. F. P., Ribeiro, J. E. S., Carvalho, T. K. N., Ribeiro, J. P. O., Guerra, N. M., ... Sousa Júnior, S. P. (2013). Local Botanical Knowledge About Useful Species in a Semi-Arid Region From Northeastern Brazil. Gaia Scientia, 7(1), 80-103.

Sousa, R. F., Silva, R. A. R., Rocha, T. G. F., Santana, J. A. S., \& Vieira, F. A. (2015). Etnoecologia e etnobotânica da palmeira carnaúba no semiárido Brasileiro. Cerne, 21(4), 587-594. https://doi.org/10.1590/01047760201521041764

Souza, A. dos S., Albuquerque, U. P., Nascimento, A. L. B. do, Santoro, F. R., Torres-Avilez, W. M., Lucena, R. F. P. de, ... Monteiro, J. M. (2017). Temporal evaluation of the Conservation Priority Index for medicinal plants. Acta Botanica Brasilica, 31(2), 169179. https://doi.org/10.1590/0102-33062017abb0027

Torres, R. R., Lapola, D. M., \& Gamarra, N. L. R. (2017). Future climate change in the caatinga. In J. M. C. Silva, I. R. Leal, \& M. Tabarelli (Eds.), Caatinga: The Largest Tropical dry forest region in South America (pp. 383-410). Springer International Publishing. 